\title{
Toll-like receptors 2 expression in mediastinal lymph node of patients with sarcoidosis
}

\author{
Xianqiu Chen ${ }^{1 \#}$, Deping Zhao ${ }^{2 \#}$, Ye Ning ${ }^{2 \#}$, Ying Zhou ${ }^{3} \wedge$ \\ ${ }^{1}$ Department of Respiratory Medicine, Shanghai Pulmonary Hospital, Tongji University School of Medicine, Shanghai, China; ${ }^{2}$ Department of \\ Thoracic Surgery, Shanghai Pulmonary Hospital, Tongji University School of Medicine, Shanghai, China; ${ }^{3}$ Department of Respiratory Medicine and \\ Clinical Research Center, Shanghai Pulmonary Hospital, Tongji University School of Medicine, Shanghai, China \\ Contributions: (I) Conception and design: Y Zhou; (II) Administrative support: Y Zhou; (III) Provision of study materials or patients: All authors; (IV) \\ Collection and assembly of data: All authors; (V) Data analysis and interpretation: X Chen, D Zhao, Y Ning; (VI) Manuscript writing: All authors; (VII) \\ Final approval of the manuscript: All authors. \\ "These authors contributed equally to this work. \\ Correspondence to: Ying Zhou, MD. Department of Respiratory Medicine and Clinical Research Center, Shanghai Pulmonary Hospital, Tongji \\ University School of Medicine, 507 Zhengmin Road, Shanghai, China. Email: docyingzhou@163.com.
}

Background: Toll-like receptors (TLRs) play a vital role as a first defense mechanism linking the innate with the adaptive immune system. Prior studies showed that TLR2 participated in immune responses of sarcoidosis. However, the role of TLR2 in the progression of mediastinal lymph nodes associated with sarcoidosis is still unknown. The current study aims to investigate the expression of Toll-like receptors 2 (TLR2) in mediastinal lymph nodes of patients with sarcoidosis.

Methods: Mediastinal lymph nodes biopsy specimens were collected from 10 patients with sarcoidosis and 11 normal controls. The expression of TLR2 in mediastinal lymph nodes was detected by immunohistochemistry.

Results: In mediastinal lymph nodes specimens, immunohistochemical examination revealed that expression of TLR2 could be detected in sarcoidosis patients, while it was scarcely detected in the mediastinal lymph nodes of control. The mean optical density of TLR2 in mediastinal lymph nodes of sarcoidosis was significantly higher than controls $(124.9 \pm 24.3$ vs. 92.6 $\pm 35.2, \mathrm{P}=0.026)$. Among patients with sarcoidosis, correlation analysis showed that the mean optical density of TLR2 in mediastinal lymph nodes positively correlated with the level of 24-hour urinary calcium $(\mathrm{R}=0.781, \mathrm{P}=0.038)$.

Conclusions: The expression of TLR2 was upregulated in mediastinal lymph nodes of sarcoidosis patients. The expression of TLR2 in mediastinal lymph nodes was associated with the level of 24-hour urinary calcium, suggesting that TLR2 might become another predictor of disease activity.

Keywords: Toll-like receptor 2 (TLR2); sarcoidosis; mediastinal lymph node

Submitted Aug 04, 2020. Accepted for publication Sep 11, 2020.

doi: 10.21037/atm-20-6103

View this article at: http://dx.doi.org/10.21037/atm-20-6103

\section{Introduction}

Sarcoidosis is a multisystem disorder characterized by non-caseating granuloma formation, most often affecting the lungs and lymph nodes (1). Although the etiology of sarcoidosis remains unknown, the isolation of Propionibacterium acnes (P. acnes) by bacterial culture and the amount of $P$. acnes RNA detected by a quantitative real-time polymerase chain reaction in sarcoid lymph nodes suggests

$\wedge$ ORCID: 0000-0002-5922-1663. 
a close link between infection and sarcoidosis $(2,3)$.

Toll-like receptors (TLRs), which are crucial in the innate immune response to invading pathogens through the recognition of conserved pathogen-associated molecular patterns, play an essential role as a first defense mechanism linking the innate with the adaptive immune system (4). Bacteria play a role in sarcoidosis pathogenesis; TLRs may be involved in the initiation of a first immune reaction. There is increasing evidence that TLRs activation could worsen autoimmunity in sarcoidosis. TLR2 can recognize and combine with the pathogen associated molecular model antigen of gram-positive bacteria, then activate downstream signal transduction through toll/IL-1 receptor (TIR) domain, resulting in increased secretion of immunomodulatory factors, up-regulation of costimulatory molecules and antigen, and triggering adaptive immune response $(5,6)$.

TLRs comprise a family of at least 11 known members in humans. Of the several identified TLRs, TLR2 participated in immune responses of sarcoidosis. Prior studies showed that TLR2 agonists, including Staphylococcus aureus peptidoglycan or synthetic TLR2 ligands, stimulate cytokine production in BAL cells from patients with sarcoidosis $(7,8)$. In the process of sarcoidosis granuloma formation, TLR2 can regulate the pro-inflammatory cytokines by pulmonary macrophages, and the granulomatous inflammatory response was reduced in TLR2 gene knockout mice (8-10).

However, the role of TLR2 in the progression of mediastinal lymph nodes associated with sarcoidosis is still mostly unknown. Local expression of TLR2 at sites of inflammation is still to be investigated in sarcoidosis. This study aimed to investigate the expression of TLR2 in mediastinal lymph nodes of patients with sarcoidosis.

We present the following article in accordance with the MDAR reporting checklist (available at http://dx.doi. org/10.21037/atm-20-6103).

\section{Methods}

\section{Study subjects}

Mediastinal lymph nodes, biopsy specimens from 10 patients with sarcoidosis, diagnosed at Shanghai Pulmonary Hospital from May 2018 to October 2019, were collected in this study. Eleven normal mediastinal lymph node biopsy specimens from patients with lung carcinoma in situ were used as normal controls. All mediastinal lymph nodes biopsy specimens are retrieved by mediastinoscopy or thoracoscopy surgery to confirm the clinical diagnosis. The diagnosis of sarcoidosis was established with clinical symptoms, radiological features, and biopsy evidence of non-caseating epithelioid cell granulomas after exclusion of other known causes of granulomatosis, as defined by the American Thoracic Society (ATS)/ European Respiratory Society consensus statement on sarcoidosis (1). Demographic characteristics, serological tests, and lung function data were collected from an electronic database. The $\mathrm{X}$-ray stage of sarcoidosis was categorized according to the Scadding scoring system (11). Stage 0 describes no visible intrathoracic findings. Stage I is bilateral hilar lymphadenopathy, which may be accompanied by paratracheal adenopathy. Stage II is a bilateral hilar adenopathy accompanied by parenchymal infiltration. Stage III is parenchymal infiltration without hilar adenopathy. Stage IV comprises advanced fibrosis with evidence of honeycombing, hilar retraction, bullae, cysts, and emphysema. Phenotypic organ involvement was confirmed for each patient by using the revised WASOG criteria described in 2014 (12). The study was conducted in accordance with the Declaration of Helsinki (as revised in 2013). The ethics committee approved the research of Shanghai Pulmonary Hospital (No. K20-072), and informed consent was taken from all the participants.

\section{The detection of TLR2 expression by immunobistochemistry}

All the specimens were fixed in $4 \%$ neutral buffered formalin, processed routinely, and embedded in paraffin, sectioned continuously, with a thickness of $4 \mu \mathrm{m}$, and baked at $60{ }^{\circ} \mathrm{C}$ for 60 minutes. For further investigation, sections were stained with hematoxylin-eosin, acid-fast stain, and methenamine silver stain. Immunohistochemical staining was performed for TLR-2. After conventional dehydration, antigen repair was performed using ethylenediaminetetraacetic acid (EDTA). $10 \mathrm{~mL}$ distilled water was added, $5 \mathrm{~mL} 10 \%$ hydrogen peroxide was added and incubated at room temperature for 30 minutes. A phosphate-buffered solution (PBS) was washed three times, 3 minutes each. The monoclonal antibody of TLR2 (1:100, purchased from Roche) was incubated at $37^{\circ} \mathrm{C}$ for 60 minutes, and washed with PBS for three times, each time for 3 minutes.TLR2 protein labeled with horseradish peroxidase (HRP) was added and incubated at $37{ }^{\circ} \mathrm{C}$ for 20 minutes. PBS was rinsed three times, 3 minutes each. After the addition of diaminobenzidine (DAB), PBS was 
Table 1 Characteristics of 10 patients with sarcoidosis

\begin{tabular}{lc}
\hline Variables & Sarcoidosis $(\mathrm{n}=10)$ \\
\hline Sex, male/female & $2 / 8$ \\
Age, year & $55.3 \pm 8.4$ \\
Smoker (yes/ex/never) & $0 / 0 / 10$ \\
X-ray stage (O/I/II/III/IV) & $0 / 3 / 7 / 0 / 0$ \\
Löfgren's syndrome & 1 \\
Organ involvement & \\
Lung & 10 \\
Non-thoracic lymph node & 5 \\
$\quad$ Liver & 1 \\
Spleen & 1 \\
Calcium & 1 \\
On oral steroid treatment (yes/no) & $1 / 9$ \\
ESR (mm/h) & $36.5 \pm 35.4$ \\
SACE (IU/L) & $54.4 \pm 52.7$ \\
slL-2 (U/mL) & $821.0 \pm 477.7$ \\
Serum calcium (mmol/L) & $2.28 \pm 0.09$ \\
24-hour urinary calcium (mmol/24 h) & $6.12 \pm 1.05$ \\
Pulmonary function tests & \\
FVC (L) & \\
FVC (\% predicted) & \\
FEV $(\mathrm{L})$ & \\
FEV $(\%$ predicted) & \\
\hline
\end{tabular}

ESR, erythrocyte sedimentation rate; SACE, serum angiotensinconverting enzyme; IL, interleukin; FVC, forced vital capacity; $\mathrm{FEV}_{1}$, forced expiratory volume in one second; DLCO, diffusing capacity of the lung for carbon monoxide.

rinsed and dyed. After dehydration, it was observed under a microscope. The staining of TLR-2 was evaluated by the Image-Pro Plus analysis software version 6.0. Positive signals are quantified as the mean optical density (integrated option density/area). This procedure was repeated twice and average value of mean optical density was selected.

\section{Statistical analysis}

SPSS (version 26, IBM) and GraphPad Prism (version 8 ) were used for statistical analysis and drawing graphs, respectively. Qualitative data are presented as mean \pm standard deviation (SD). The independent sample student's $t$-test was used for comparisons between SA and HD groups. The association between two continuous variables was analyzed using Pearson's correlation (for two parametric variables) or Spearman's rank correlation (for two nonparametric variables or one nonparametric variable with one parametric variable). A value of $\mathrm{P}<0.05$ was considered statistically significant.

\section{Results}

\section{Characteristics of study subjects}

Demographic characteristics, serological tests, and lung function data of all ten sarcoidosis patients were shown in Table 1. Among the 10 patients with sarcoidosis, 2 were males, and 8 were females. The youngest patient was 42 years of age, and the oldest patient was 67 years of age, with an average age of $55.3 \pm 8.4$ years. According to the Scadding X-ray scoring system, 3 of 10 patients were classified as stage I. The other 7 patients were classified as stage II. Although lung was the most involved organ, many patients exhibited extrapulmonary manifestations. Among the 11 control patients, 6 were males, and 5 were females with an average age of $53.4 \pm 10.4$ years. In the control group, 3 were males, and 7 were females, with an average age of $50.2 \pm 7.9$ years. There was no significant difference between gender or age in two groups.

\section{Histologic diagnosis}

Biopsy specimens were diagnosed as non-caseating epithelioid cell granulomas in all ten sarcoidosis patients. Other known causes of granulomatosis were excluded by histologic features, with negative acid-fast stain and methenamine silver stain.

The CT scan presentation and histology of an individual patient with stage I sarcoidosis are shown in Figure 1. CT scan showed mediastinal and hilar lymphadenopathy, and biopsy histopathology showed non-caseating epithelioid cell granulomas. Histology shows typical structure or reactive hyperplasia of mediastinal lymph nodes in 11 controls.

\section{Immunobistochemistry for TLR2}

The expression of TLR2 in the mediastinal lymph nodes of patients with sarcoidosis and immunohistochemical 

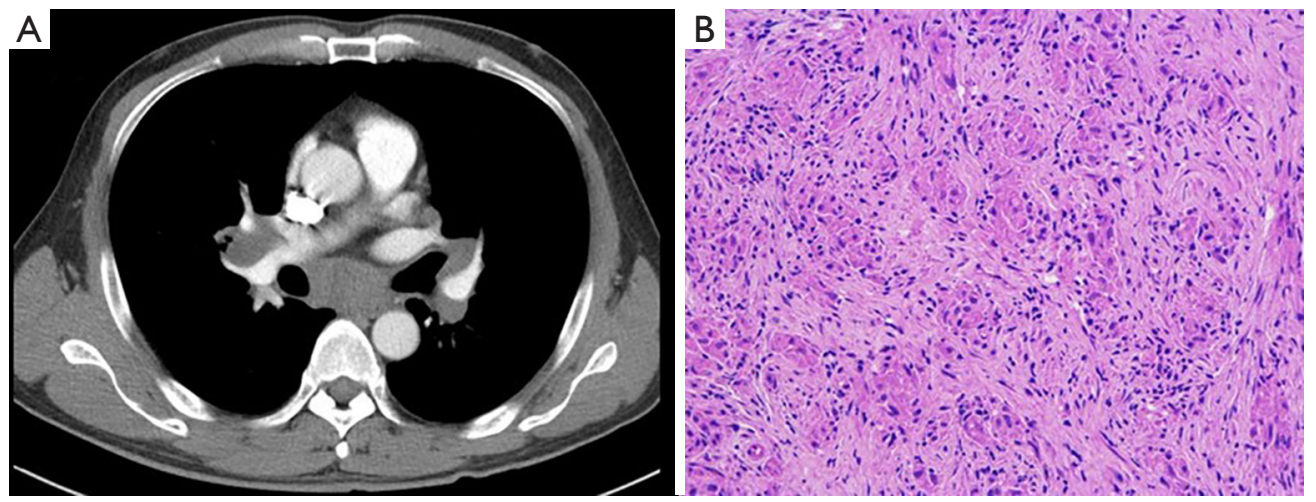

Figure 1 CT scan presentation and histologic features of sarcoidosis patients. (A) CT scan showed mediastinal and hilar lymphadenopathy in a patient with sarcoidosis; (B) biopsy histopathology with hematoxylin-eosin staining showed non-caseating epithelioid cell granulomas in a patient with sarcoidosis $(\times 100)$.

method detected the control group. In mediastinal lymph nodes specimens with sarcoidosis, immunohistochemical examination revealed that expression of TLR-2 was detected, while TLR-2 was scanty in the lymph nodes of normal controls (Figure 2). The tan color is a positive result of immunohistochemistry. It was visually observed that the patient with sarcoidosis has more TLR2 positive results than control. The mean optical density of TLR2 in patients with sarcoidosis was significantly higher than that in normal controls $(124.9 \pm 24.3$ vs. 92.6 $\pm 35.2, \mathrm{P}=0.026)$ (Figure 3).

\section{The correlations between the TLR2 and clinical data}

Among the patients with sarcoidosis, correlation analysis showed that the mean optical density of TLR2 in mediastinal lymph nodes has a positive correlation trend with the disease activity index (SACE, serum, calcium, and ESR), and has a negative correlation trend with lung function (Table 2). There were 7 of 10 patients who took the 24-hour urinary calcium test. Correlation analysis showed the 24-hour urinary calcium has a significant positive correlation with the mean optical density of TLR2 in these seven patients with sarcoidosis ( $\mathrm{r}=0.781, \mathrm{P}=0.038$, Figure 4).

\section{Discussion}

TLRs play a crucial role in innate immune activation and regulation through recognition of pathogen-associated molecular patterns and endogenous peptides and induce antigen-presenting cells to secrete IL-12 and IL-18, which stimulates naïve $\mathrm{T}$ cells to become Th1 cells $(13,14)$. In immune system diseases, TLR2 participates in the initiation and maintenance of immune response, which depends on the signal transduction pathway of myeloid differentiation factor 88 (MyD88) (15). When the body is stimulated by exogenous molecules, TLR2 receptor protein on the APC surface recognizes the ligand and dimers, and then it is activated to recruit the downstream signal protein MyD88. Its TIR domain interacts with the death domain of MyD88 and recruits IL-1R associated kinase 4 (IL-1R associated kinase 4), IRAK-4 is located around TLR2, leading to the activation and autophosphorylation of IRAK-4, activation of IL-1R associated kinase 1 (IRAK-1), and then to tumor necrosis factor receptor-6 (TNF- $\alpha$ ), TRAF-6 interacts with and activates a variety of mitogen binding protein kinases, and then activates nuclear factor kappa-B (NF-kb), resulting in the expression of inflammatory factors and chemokines CXCL8 and CCL20 (16-18). Granulomatous inflammation in sarcoidosis has been showed to be a Th1-mediated response (19). The increased TLR expression found in sarcoidosis could lead to later Th1 cell proliferation and cause the granuloma formation. Among the several TLRs, TLR2 was found to be associated closely with the development of sarcoidosis $(9,10)$. Current theories also propose $\mathrm{P}$. acnes, the known cause of acne vulgaris, could be responsible for the pathogenesis of sarcoidosis $(3,20)$. The inflammatory response to $\mathrm{P}$. acnes in acne vulgaris is known to be mediated through TLR 2 (21). However, the role of TLR2 in tissue injury of sarcoidosis is not yet clear. Data on TLR2 expression in mediastinal lymph nodes of sarcoidosis are lacking. Therefore, the current study aimed to characterize the expression of TLR-2 in Mediastinal lymph nodes of patients with sarcoidosis.

In the present study, we observed remarkable expression 


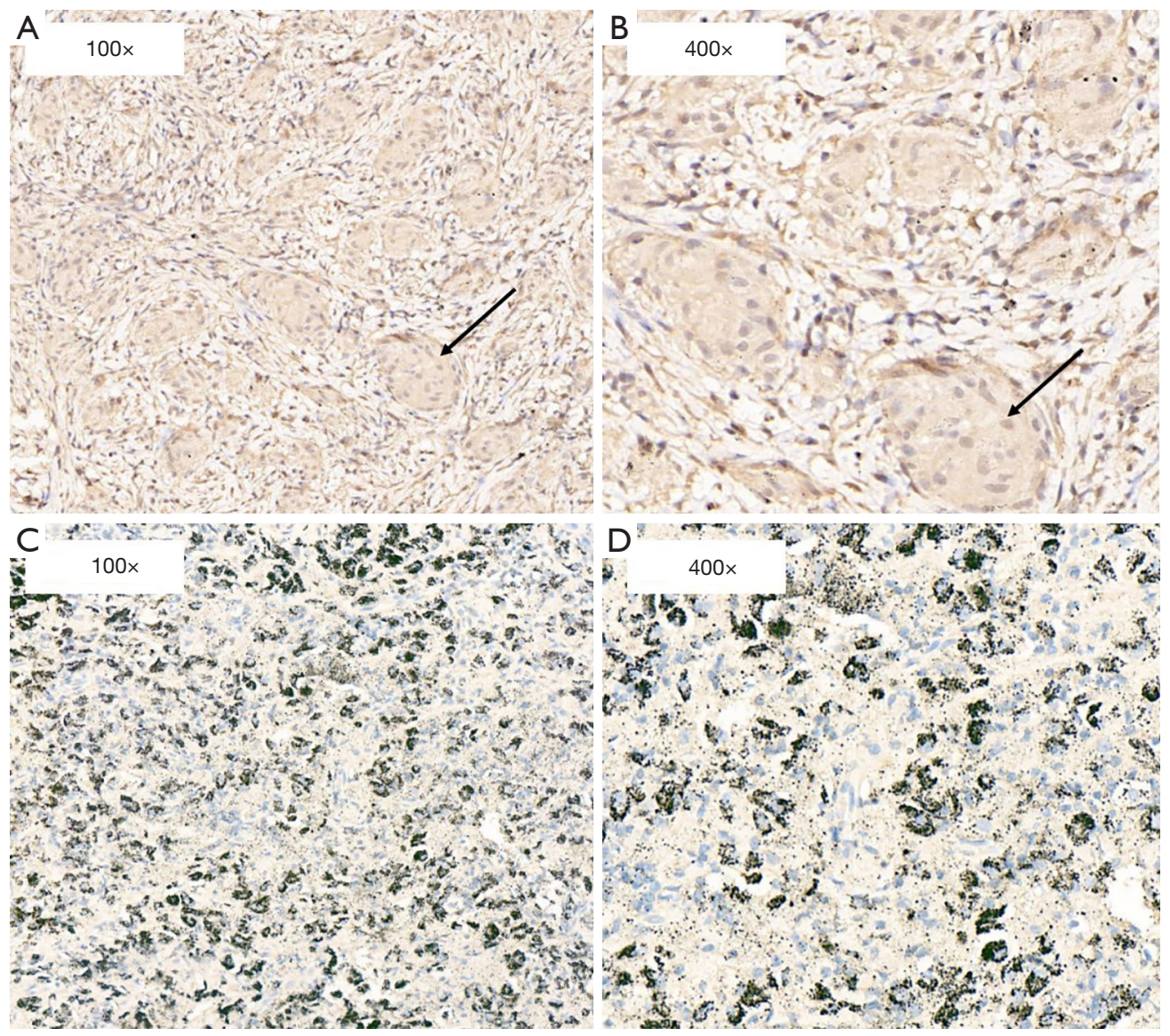

Figure 2 Immunohistochemistry staining for TLR2 in the mediastinal lymph node specimens. (A) and (B) Immunohistochemical staining of TLR-2 in the mediastinal lymph nodes of patients with sarcoidosis $(\times 100$ and $\times 400)$ (marked with arrows). (C) and (D) Immunohistochemical staining of TLR-2 in the mediastinal lymph nodes of control $(\times 100$ and $\times 400)$.

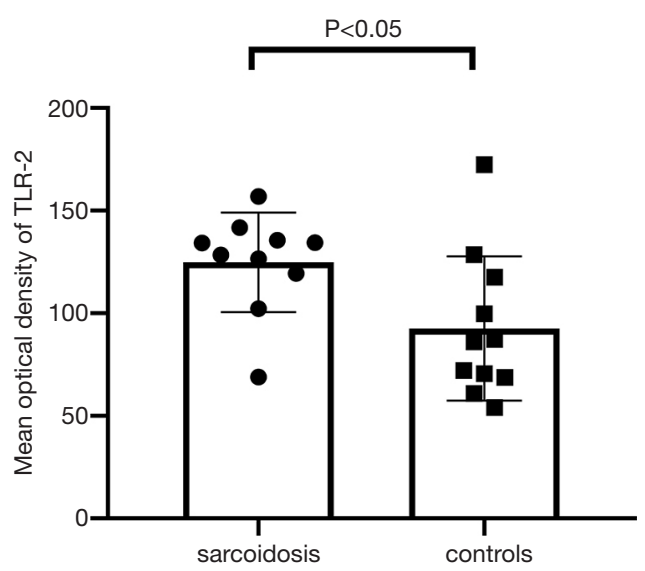

Figure 3 The mean optical density of TLR2 in mediastinal lymph nodes of patients with sarcoidosis and the controls.
Table 2 Correlation analysis between TLR2 and clinical data

\begin{tabular}{|c|c|c|}
\hline Clinical data & $\mathrm{R}$ & $P$ value \\
\hline SACE & 0.433 & 0.284 \\
\hline Serum calcium & 0.199 & 0.582 \\
\hline 24-hour urinary calcium & 0.781 & 0.038 \\
\hline ESR & 0.009 & 0.986 \\
\hline sIL-2 & -0.440 & 0.383 \\
\hline FVC (\% predicted) & -0.038 & 0.930 \\
\hline $\mathrm{FEV}_{1}(\%$ predicted $)$ & -0.200 & 0.635 \\
\hline DLCO (\% predicted) & -0.162 & 0.794 \\
\hline
\end{tabular}

The data presented in italic type are statistically significant. SACE, serum angiotensin-converting enzyme; ESR, erythrocyte sedimentation rate; IL, interleukin; FVC, forced vital capacity; $\mathrm{FEV}_{1}$, forced expiratory volume in one second; DLCO, diffusing capacity of the lung for carbon monoxide. 


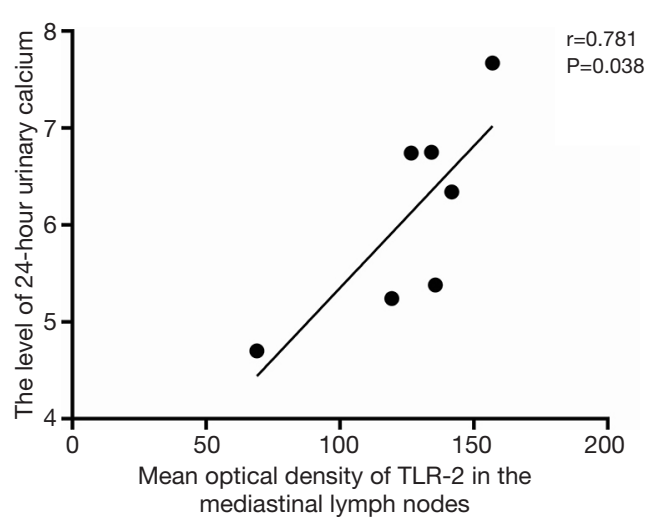

Figure 4 Correlation between the mean optical density of TLR2 in the mediastinal lymph nodes and the 24-hour urinary calcium in 7 sarcoidosis patients.

of TLR2 in mediastinal lymph node specimens from sarcoidosis patients, while patients with lung carcinoma in situ showed weak expression of TLR2 in mediastinal lymph nodes. These results were consistent with the studies by Chen and Gabrilovich et al., which showed the expression of TLR2 in macrophages of bronchoalveolar lavage fluid in patients with sarcoidosis is significantly increased compared with the control group $(9,10)$. After the activation of TLR2, cytokines, and antibodies produced by peripheral blood mononuclear cells are dysregulated, resulting in increased secretion of inflammatory cytokines $(9,10)$. Another study showed TLR-2 expression is upregulated in different peripheral blood immune cells in sarcoidosis, and induction of TLR-2 expression was found not only in Tregs but also in the heterogeneous population of PBMC of patients with pulmonary sarcoidosis (22). In Reyes' study of PBMC gene expression profiles, after induction with $M$. tuberculosis also increased, the expression of TLR-2 was found in sarcoidosis (23). These studies proposed the upregulation of TLR2 in peripheral blood or bronchoalveolar lavage fluid of patients with sarcoidosis. To the best of our knowledge, this is the first study to characterize the increased expression of TLR2 in mediastinal lymph nodes of patients with sarcoidosis by immunohistochemistry, which showed that local expression of TLR2 in mediastinal lymph nodes might be involved in the development of sarcoidosis. The dysregulation of the expression level of TLR2 warrants further investigation of its function on the potential causative agent and pathogenic mechanism of pulmonary sarcoidosis.

Altered calcium metabolism, which affects approximately
$5 \%$ to $25 \%$ of sarcoidosis patients, can present as hypercalcemia, hypercalciuria, or kidney stones and have been considered correlating with disease activity (24-26). Calcium metabolism alteration in sarcoidosis is usually because of increased activity of 1-alpha hydroxylase in macrophages of pulmonary granuloma, resulting in low levels of 25-hydroxyvitamin D and high levels of calcitriol (25). As mentioned before, TLR2 is an important receptor for recognition of bacterial antigen and is involved in the immune process of sarcoidosis granuloma formation. In this study, it was found 24 hours after urinary calcium level was positively correlated with the TLR2 level, suggesting that the level of TLR2 might be directly related to disease activity, which also suggests TLR2 might become another predictor of disease activity.

There are some limitations to this study. The number size of patients in this study was small. More extensive studies are called for to be further verification of TLR2 function in sarcoidosis in the later study. Second, the mechanism of the TLR2 signaling pathway in the etiology and development of sarcoidosis needs to be intensively studied. Meanwhile, double immunofluorescence of TLR2 should be performed to determine which cell types displayed increased TLR2 expression in future research.

In conclusion, the expression of TLR2 was upregulated in mediastinal lymph nodes of patients with sarcoidosis. The expression of TLR2 has a positive correlation between urinary calcium levels, suggesting TLR2 may be an indicator for predicting sarcoidosis activity.

\section{Acknowledgments}

This work was supported by grants from National Science Foundation of Shanghai, China [No. 18ZR1431400].

\section{Footnote}

Reporting Checklist: The authors have completed the MDAR reporting checklist. Available at http://dx.doi.org/10.21037/ atm-20-6103

Data Sharing Statement: Available at http://dx.doi. org/10.21037/atm-20-6103

Conflicts of Interest: All authors have completed the ICMJE uniform disclosure form (available at http://dx.doi. org/10.21037/atm-20-6103). The authors have no conflicts of interest to declare. 
Ethical Statement: The authors are accountable for all aspects of the work in ensuring that questions related to the accuracy or integrity of any part of the work are appropriately investigated and resolved. The study was conducted in accordance with the Declaration of Helsinki (as revised in 2013). The institutional ethics committee of Shanghai Pulmonary Hospital approved the study (No. K20-072), and informed consent was taken from all the participants.

Open Access Statement: This is an Open Access article distributed in accordance with the Creative Commons Attribution-NonCommercial-NoDerivs 4.0 International License (CC BY-NC-ND 4.0), which permits the noncommercial replication and distribution of the article with the strict proviso that no changes or edits are made and the original work is properly cited (including links to both the formal publication through the relevant DOI and the license). See: https://creativecommons.org/licenses/by-nc-nd/4.0/.

\section{References}

1. Lee HJ, Seo H, Cha SI, et al. Sarcoidosis presenting pulmonary subsolid nodules that mimic lung adenocarcinoma in a patient with history of uveitis and arrhythmia: a case report. Ann Transl Med 2019;7:496.

2. Abe C, Iwai K, Mikami R, et al. Frequent isolation of Propionibacterium acnes from sarcoidosis lymph nodes. Zentralbl Bakteriol Mikrobiol Hyg A 1984;256:541-7.

3. Novosadova E, Navratilova Z, Ordeltova M, et al. Comparison of lymphocyte immune phenotypes in bronchoalveolar lavage of non-smoking patients with sarcoidosis and other interstitial lung diseases. J Thorac Dis 2019;11:2287-96.

4. Akira S, Uematsu S, Takeuchi O. Pathogen recognition and innate immunity. Cell. 2006;124:783-801.

5. Tartey S, Takeuchi O. Pathogen recognition and Toll-like receptor targeted therapeutics in innate immune cells. Int Rev Immunol 2017;36:57-73.

6. Gambero M, Teixeira D, Butin L, et al. Propionibacterium acnes induces an adjuvant effect in B-1 cells and affects their phagocyte differentiation via a TLR2-mediated mechanism. Immunobiology 2016;221:1001-11.

7. Veltkamp M, Wijnen PA, van Moorsel CH, et al. Linkage between Toll-like receptor (TLR) 2 promotor and intron polymorphisms: functional effects and relevance to sarcoidosis. Clin Exp Immunol 2007;149:453-62.

8. Wikén M, Grunewald J, Eklund A, et al. Higher monocyte expression of TLR2 and TLR4, and enhanced proinflammatory synergy of TLR2 with NOD2 stimulation in sarcoidosis. J Clin Immunol 2009;29:78-89.

9. Chen ES, Song Z, Willett MH, et al. Serum amyloid A regulates granulomatous inflammation in sarcoidosis through Toll-like receptor-2. Am J Respir Crit Care Med 2010;181:360-73.

10. Gabrilovich MI, Walrath J, van Lunteren J, et al. Disordered Toll-like receptor 2 responses in the pathogenesis of pulmonary sarcoidosis. Clin Exp Immunol 2013;173:512-22.

11. Scadding JG. Prognosis of intrathoracic sarcoidosis in England. A review of 136 cases after five years' observation. Br Med J 1961;2:1165-72.

12. Judson MA, Costabel U, Drent M, et al. The WASOG Sarcoidosis Organ Assessment Instrument: An update of a previous clinical tool. Sarcoidosis Vasc Diffuse Lung Dis 2014;31:19-27.

13. Akira S, Takeda K, Kaisho T. Toll-like receptors: critical proteins linking innate and acquired immunity. Nat Immunol 2001;2:675-80.

14. Wagner H. Endogenous TLR ligands and autoimmunity. Adv Immunol 2006;91:159-73.

15. Liu $Y$, Yin $H$, Zhao M, et al. TLR2 and TLR4 in autoimmune diseases: a comprehensive review. Clin Rev Allergy Immunol 2014;47:136-47.

16. Avbelj M, Horvat S, Jerala R. The role of intermediary domain of MyD88 in cell activation and therapeutic inhibition of TLRs. J Immunol 2011;187:2394-404.

17. Hatao F, Yamamoto M, Muroi M, et al. MyD88-induced downregulation of IRAK-4 and its structural requirements. FEMS Immunol Med Microbiol 2008;53:260-4.

18. Muroi M, Tanamoto K. TRAF6 distinctively mediates MyD88- and IRAK-1-induced activation of NF-kappaB. J Leukoc Biol 2008;83:702-7.

19. Wahlström J, Katchar K, Wigzell H, et al. Analysis of intracellular cytokines in CD4+ and CD8+ lung and blood T cells in sarcoidosis. Am J Respir Crit Care Med 2001;163:115-21.

20. Zhao MM, Du SS, Li QH, et al. High throughput 16SrRNA gene sequencing reveals the correlation between Propionibacterium acnes and sarcoidosis. Respir Res 2017;18:28.

21. Kim J, Ochoa MT, Krutzik SR, et al. Activation of tolllike receptor 2 in acne triggers inflammatory cytokine responses. J Immunol 2002;169:1535-41.

22. Kachamakova-Trojanowska N, Jazwa-Kusior A, Szade $\mathrm{K}$, et al. Molecular profiling of regulatory $\mathrm{T}$ cells in 
pulmonary sarcoidosis. J Autoimmun 2018;94:56-69.

23. Reyes N, Bettin A, Reyes I, et al. Microarray analysis of the in vitro granulomatous response to Mycobacterium tuberculosis H37Ra. Colomb Med (Cali) 2015;46:26-32.

24. Ying Z, Elyse EL, Yinping F, et al. Clinical characteristics of sarcoidosis patients in the United States versus China. Sarcoidosis Vasc Diffuse Lung Dis 2017;34:209-16.

25. Baughman RP, Papanikolaou I. Current concepts regarding calcium metabolism and bone health in sarcoidosis. Curr Opin Pulm Med 2017;23:476-81.

26. Cameli P, Gonnelli S, Bargagli E, et al. The Role of Urinary Calcium and Chitotriosidase in a Cohort of Chronic Sarcoidosis Patients. Respiration 2020;99:207-12.

(English Language Editor: J. Chapnick)
Cite this article as: Chen X, Zhao D, Ning Y, Zhou Y. Tolllike receptors 2 expression in mediastinal lymph node of patients with sarcoidosis. Ann Transl Med 2020;8(18):1182. doi: 10.21037/atm-20-6103 